\section{The Value of a Streamlined Surveillance Method}

\section{To the Editor:}

Following adoption of the streamlined surveillance method described in "Abbreviated Surveillance of Nosocomial Urinary Tract Infections: A New Approach," we noted a substantial increase over the next 11 months in both the absolute number of UTIs, as well as UTI rates (calculated per 1,000 patient days). Concerned that this increase might be an artifact produced by the new surveillance system, we examined the overestimation of this method at our facility for the month of December 1985.

Of the 48 positive cultures identified by the abbreviated method, traditional surveillance identified 45 as being true nosocomial infections. This represents an overestimation of $6 \%$, somewhat less than the $12 \%$ reported by Costel et al. We did not evaluate possible underestimation.

While our comparison of the two methods did not explain the increased UTI rate at our institution, it does support the findings of Costel et al. The overestimation inherent in the abbreviated method may be even less than the $12 \%$ reported by those authors. We enthusiastically endorse the streamlined technique because of its time-saving features, and of fer this experience as further validation of its specificity.

\section{REFERENCES}

1. Costel EE, Mitchell S, Kaiser AB: Abbreviated surveillance of nosocomial urinary tract infections: A new approach. Infect Control 1985; 6:11-13.

Susan Page, SM(ASCP) Philip Mead, MD Kathryn Howe, RN, CIC Medical Center Hospital of Vermont Burlington, Vermont

\section{Administration and Tracheostomy Care in the Home}

\section{To the Editor:}

I read with interest the inquiry in Infection Control August 1985, page 299, regarding guidelines for IV Therapy infection control practices in the home.

Ms. Crow responded by stating there are no national organizations that have addressed this issue. I would like to advise your readers of the National Intravenous Therapy Association (NITA) standards for IV Therapy which include infection control practices and home care.

NITA is a national organization representing over 3,500 Registered Nurses who are actively involved in the practice of IV Therapy, many of whom practice totally in the home care setting. Many institutions and agencies base their IV Policy and Procedure on the standards of NITA.

I would like to point out that IV tubings need to be changed at 24 to 48 hour intervals and not 48 to 72 hours as advised. The standard of 24 to 48 hours was established by NITA and is in accordance with the Centers for Disease Control (CDC) Guidelines.

Copies of the above mentioned standards may be obtained by writing to the NITA office at 87 Blanchard Road, Cambridge, MA 02138. Major standard revisions are projected to be published during 1986.

Sue Thomson, CRNI

NITA Sig Committee Chairperson on Home Health Care IV Therapy Coordinator

Gettysburg Hospital Gettysburg, Pennsylvania

Ms. Crow responds to Ms. Thomson's comments.

It is true that NITA has general guidelines for IV care in the home situation. I look forward to the revisions since more specific infection control issues need to be addressed for this rapidly expanding area.

The NITA recommendations you referred to state, "IV admixture sets should be changed every 24 hours or after each IV medication treatment." Personally I do not believe that this is practical in today's healthcare world. In fact, there are studies showing that 48-hour change is safe practice. One study, at the New England Medical Center in Boston, even shows that a 72-hour change is safe. It is interesting to note that with the advent of cost containment, many hospitals have begun to change IV sets every 72 hours with no increased risk in infection rates.

Recommendations from organizations such as NITA and the Centers for Disease Control should be reviewed when establishing any patient care practice. However, we must recognize that we live in the real world of cost containment. Consequently, we must make patient care decisions based on studies when available, and common sense when there are no good studies.

Sue Crow, RN, MSN, CIC Nurse Epidemiologist Louisiana State University Shreveport, Louisiana

\section{Correction Noted}

\section{To the Editor}

Our article "Nosocomial Fungal Infection During Hospital Renovation" in Infection Control 6(7):278-282 contains an error. On page 279 , column 1, line 29; Rhizopus indicus should in fact be Mucor indicus. I apologize for the inconvenience.

Bruce A. Hanna, PhD

Director, Clinical Microbiology Bellevue Hospital New York, New York 